\title{
Validez de constructo del Inventario de Depresión de Beck II para adolescentes
}

\author{
Construct validity of the Beck Depression Inventory II in adolescents
}

\author{
José Alfredo Contreras-Valdez \\ Laura Hernández-Guzmán \\ Miguel-Ángel Freyre \\ Facultad de Psicología, Universidad Nacional Autónoma de México
}

(Rec: 25 febrero 2015 / Acept: 30 septiembre 2015)

\begin{abstract}
Resumen
Son pocos los estudios que han analizado el modelo teórico de la depresión mediante el Inventario de Depresión de Beck II (BDI-II) en adolescentes hispanohablantes. Esta investigación se planteó dos propósitos: 1) indagar el ajuste de dos modelos factoriales en adolescentes mexicanos de población general del BDI-II, el unidimensional y el original de Beck et al. (1996) que propone los factores cognitivo-afectivo y somáticovegetativo y 2) examinar la invarianza entre sexos del modelo con mejor ajuste. Participaron en dos estudios 1010 y 610 adolescentes, respectivamente, quienes cumplimentaron el BDI-II. Mediante AFC se sometió a prueba las dos estructuras factoriales y, por medio de AFC multigrupo, se realizó el análisis de invarianza entre sexos. Los resultados revelaron la superioridad del modelo de dos factores en ambos estudios, sin hallar diferencias entre mujeres y hombres. Estos hallazgos arrojan evidencia en favor de la teoría de la depresión de Beck y de la validez de constructo del BDI-II en adolescentes.

Palabras clave: afecto, pubertad, tristeza, auto-informe y emociones.
\end{abstract}

\begin{abstract}
Few studies have analyzed theoretical model of depression through the Beck Depression Inventory II (BDIII) in Spanish-speaking adolescents. This research had two aims: 1) to know the fit of two BDI-II factor models in community Mexican adolescents, the one-dimension model and the Beck et al. (1996)'s original model posing the Cognitive-Affective and Somatic-Vegetative factors, and 2) to examine model invariance across sexes with the best-fitting model. In two studies, 1010 and 610 adolescents participated, respectively, who completed the BDI-II. Through CFA the two factor structures were tested and, by means of multi-group CFA, invariance analysis across sexes was performed. Results revealed superiority of the two-factor model in both studies, without differences between women and men. These findings lead to evidence for Beck's theory of depression and construct validity of the BDI-II in adolescents.

Keywords: affect, puberty, sadness, self-report, emotions.
\end{abstract}

\footnotetext{
Esta investigación fue posible gracias al financiamiento otorgado a la Dra. Laura Hernández-Guzmán, responsable de los proyectos PAPIIT IN305512 e IN305814, Dirección General de Asuntos del Personal Académico de la UNAM.

Agradecimientos: Se agradecen las facilidades brindadas por las autoridades y personal de las escuelas participantes, así como el apoyo de los siguientes estudiantes en el reclutamiento de las escuelas y la recolección de datos: Idalia Aragón, Katya Franco, Edith García, Berenice González, Brenda López, Carmen Pompa, Joselyn Rodríguez, Rodrigo Rosales, Emmanuel Ruiz, Marbella Sánchez, Victoria Sánchez y Jessica Téllez.

Correspondencia: relacionada con este artículo debe dirigirse a la Dra. Laura Hernández Guzmán, Facultad de Psicología, Universidad Nacional Autónoma de México. Avenida Universidad No. 3004, Edif. E, cubículo 209, Col. Copilco-Universidad, C.P. 04510, Delegación Coyoacán, México, D.F. Tel. (55) 5622-2250. E-mail: 1her@unam.mx
} 


\section{Introducción}

La depresión es un trastorno del estado de ánimo muy frecuente que afecta al individuo en los diversos contextos en los que participa (Ezquiaga, García, Díaz y García, 2011). Actualmente se sabe que más de 350 millones de personas en el mundo padecen depresión y que ésta contribuye de forma importante a la carga de morbilidad a nivel internacional (Organización Mundial de la Salud, 2012). La depresión no sólo destaca por su elevada prevalencia, sino también por la probabilidad de recaída y recurrencia asociada (Zauszniewski y Rong, 1999). Una contrariedad más es el elevado coste económico que implica, el cual se traduce en baja productividad, ausentismo laboral, cuidados ambulatorios, hospitalizaciones y tratamientos farmacológicos (Valladares, Dilla y Sacristán, 2009).

Se ha encontrado que el primer episodio de depresión o el incremento exacerbado de síntomas depresivos ocurre generalmente cuando el individuo es adolescente, por ser esta etapa del desarrollo donde los problemas psicológicos del individuo aumentan (Hankin, 2006). Es también en la adolescencia cuando se acentúan las diferencias entre sexos, ya que las mujeres presentan más síntomas que los hombres (Araya, Montero-Marín, Barroilhet, Fritsch, Gaete y Montgomery, 2013; Benjet et al. 2009; Del Barrio, Mestre, Tur y Samper, 2004; Uslu, Kapci, Oncu, Ugurlu y Turkcapar, 2008). Empero, si bien las diferencias en intensidad son comunes, algunos investigadores recomiendan el análisis de invarianza para conocer también las posibles discrepancias cualitativas. En culturas orientales se ha hallado diferencias entre mujeres y hombres en relación con la estructura latente de la depresión (Wu, 2010a), no así en adolescentes latinoamericanos (Araya et al. 2013), por lo que resulta necesario arrojar más luz sobre este tema.

La depresión de los adolescentes se asocia con otras variables psicológicas como ansiedad y afecto negativo (Osman, Downs, Barrios, Kopper, Gutierrez y Chiros, 1997; Sandín, 2003), baja autoestima (Del Barrio et al. 2004), rumia cognitiva (Beltrán, Freyre y Hernández-Guzmán, 2012), trastornos de personalidad y conducta agresiva (Agudelo, Spielberg y Buela-Casal, 2007; Hankin, 2006), etc. A largo plazo predice estrés, abuso de sustancias y discapacidad laboral (Keenan-Miller, Hammen y Brennan, 2007), bajos niveles de apoyo social (Naicker, Galambos, Zeng, Senthilselvan y Colman, 2013) y síntomas de depresión en la edad adulta (Benjet, Borges, Medina-Mora, Fleiz-Bautista y Zambrano-Ruiz, 2004).
Distintos paradigmas han ofrecido explicaciones sobre la depresión, como la teoría de la desesperanza (Abramson, Seligman y Teasdale, 1978) o los estilos de respuesta (NolenHoeksema, 1991), entre otros. No obstante, la propuesta de Beck es especialmente relevante debido al sustento empírico del que goza, proporcionado por la eficacia comprobada de las intervenciones clínicas basadas en sus supuestos teóricos, dirigidas tanto a población clínica como general, así como a adultos y adolescentes (Butler, Chapman, Forman y Beck, 2006; Clark y Beck, 2010; DeRubeis et al., 2005; Dobson et al. 2008; Lakdawalla, Hankin y Mermelstein, 2007).

De acuerdo con Beck (1967), la depresión se caracteriza por tristeza, apatía, concepto negativo de sí mismo, autorreproches, culpa, conductas de evitación, síntomas somáticos y cambios en el nivel de actividad física. Los síntomas se originan a partir de esquemas cognitivos desadaptados sobre sí mismo, el mundo y el futuro, los cuales son rígidos y absolutistas. La prevalencia de estos esquemas interfiere con el procesamiento de la información positiva y produce pensamientos automáticos negativos y sesgados que traen como desenlace problemas emocionales. Así, se entiende que el estado emocional de un individuo depende de las creencias que tiene sobre sus experiencias de vida (Beck, Rush, Shaw y Emery, 1979; Clark y Beck, 2010).

Con el propósito de identificar los síntomas depresivos y cuantificar su intensidad, en 1961 surgió el Inventario de Depresión de Beck (BDI por sus siglas en inglés). El instrumento original se basó en observaciones clínicas y descripciones de pacientes psiquiátricos con depresión. No obstante, en 1979 Beck y sus colaboradores comenzaron a usar una versión modificada, el BDI-IA, que ya incorporaba seis de los nueve criterios diagnósticos establecidos en el DSM-III. Posteriormente, con el lanzamiento de la versión revisada del DSM-IV, en 1994, se hizo presente la necesidad de otro instrumento que incluyera las nuevas normas de evaluación, por ejemplo, un periodo mínimo de dos semanas con la presencia de síntomas, entre otras. La versión resultante y la más actualizada, es el BDI-II (Beck, Steer y Brown, 1996).

El estudio del BDI-II en población adolescente ha revelado tanto fortalezas como debilidades. Se ha documentado evidencia de validez convergente tras correlacionar fuertemente con medidas de depresión y ansiedad (Araya et al. 2013), desesperanza y síntomas internalizados, así como validez divergente al relacionarse débilmente con escalas de apoyo social y síntomas externalizados (VanVoorhis y Blumentritt, 2007). Distintos trabajos han informado puntos de corte que varían de acuerdo con la muestra, pero en general 
coinciden en que el BDI-II posee sensibilidad y especificidad aceptables, así como una confiabilidad adecuada que oscila entre .90 y .91 (Melipillán, Cova, Rincón y Valdivia, 2008; Osman, Barrios, Gutierrez, Williams y Bailey, 2008; Uslu et al. 2008).

En contraste, los resultados referidos a su validez de constructo no son tan estables. El modelo de dos factores, cognitivo-afectivo y somático-vegetativo, originalmente informado por Beck et al. (1996) en población general, se ha corroborado sólo parcialmente (Osman et al., 2008; Wu y Chang, 2008). Se supone que en el primer factor, Cognitivo-afectivo, habrían de agruparse reactivos como tristeza, sentimientos de culpa, autocrítica, etc. Por su parte, el factor somático-vegetativo debería incluir ítems como pérdida de energía o cambios en el patrón de sueño, entre otros. Un problema es que, aunque se ha llegado a confirmar una estructura bidimensional, los ítems que cargan en cada factor varían de un estudio a otro, dependiendo de las características de la muestra, de población general o clínica (Beck et al. 1996). En virtud de los hallazgos inconsistentes, otros investigadores han propuesto modelos más complejos de tres factores (p.ej., Byrne, Stewart y Lee, 2004), sin haber logrado demostrar la agrupación de los ítems en dimensiones teóricamente congruentes (Ward, 2006).

Otro problema referente a la validez de constructo es que algunas traducciones del BDI-II que existen en el mundo no cuentan con datos sobre su estructura factorial (Uslu et al. 2008; VanVoorhis y Blumentritt, 2007). En español, hasta donde se sabe, sólo existen dos publicaciones acerca del BDI-II que han indagado sobre su modelo teórico en adolescentes con análisis factorial confirmatorio (AFC). En ambos estudios, realizados en Chile, el ajuste de un modelo unidimensional fue similar o incluso superior a otras estructuras de más factores (halladas previamente por medio de análisis factorial exploratorio), por resultar la explicación más parsimoniosa de la sintomatología depresiva. Empero, es necesario puntualizar que ninguno de ellos sometió a prueba la estructura de dos factores de Beck et al. (1996). La falta de estudios sobre la estructura factorial del instrumento y la inconsistencia en las dimensiones en las que se agrupan los síntomas resaltan la necesidad de seguir investigando el ajuste del modelo teórico original de Beck et al. de la depresión.

Por consiguiente, esta investigación se propuso estudiar la estructura factorial de la versión en español dirigida a adolescentes del BDI-II. Particularmente, indaga en dos estudios de validación cruzada (un primer estudio y su réplica) si se confirma la estructura unidimensional hallada en Chile
(Araya et al. 2013; Melipillán et al. 2008) o el modelo de dos dimensiones de Beck et al. (1996). Adicionalmente, se planteó examinar la invarianza entre sexos del modelo que resultare con mejor ajuste. A partir de los resultados que favorecen la estructura unidimensional, según ambas investigaciones en población latinoamericana (Araya et al. 2013; Melipillán et al. 2008) y la carencia de datos que confirmen el modelo teórico original, se planteó como hipótesis que el modelo de un solo factor mostraría un mejor ajuste en el caso de los adolescentes mexicanos. Debido a que se ha encontrado que la sintomatología depresiva de mujeres y hombres de población latinoamericana es cualitativamente idéntica (Araya et al. 2013), se esperaba que no existieran diferencias en el ajuste del mejor modelo de medida respecto al sexo.

\section{Estudio 1}

\section{Método}

\section{Participantes}

La muestra inicial constó de 1,123 estudiantes (576 mujeres y 547 hombres) de educación secundaria inferior y superior de acuerdo con la Clasificación Internacional Normalizada de la Educación (Instituto de Estadística de la UNESCO, 1997). Participaron 10 escuelas, nueve pertenecían al sector público y una al sector privado. La unidad de muestreo fue el grupo escolar. Las escuelas estaban distribuidas en diversas delegaciones de la zona metropolitana de la Ciudad de México.

Dado que esta investigación se centró en la sintomatología depresiva de adolescentes, se excluyó a los alumnos menores de 13 años ( $n=52$; de acuerdo con los criterios de Beck et al. 1996) y mayores de 18 ( $n=61$; según la Organización Panamericana de la Salud, 2008). La muestra final, integrada por un total de 1,010 estudiantes (519 mujeres y 491 hombres), se dividió aleatoriamente en dos. En la muestra $1\left(n_{1}=505\right)$ participaron 238 mujeres y 267 hombres ( 252 de secundaria inferior y 253 de secundaria superior) con una edad promedio de 15.29 años $(D E=1.45)$. La muestra $2\left(n_{2}=505\right)$ constó de 281 mujeres y 224 hombres ( 265 de secundaria inferior y 240 de secundaria superior), siendo su edad promedio de 15.12 años $(D E=1.61)$. 


\section{Instrumento}

Inventario de Depresión de Beck, segunda edición (BDI-II; adaptado a la población de Argentina por Brenlla y Rodríguez, 2006), adaptado para adolescentes mexicanos (Hernández-Guzmán, 2012). Se trata de un auto-informe que consta de 21 reactivos que miden síntomas de depresión en adolescentes de 13 años en adelante y adultos. Cada reactivo está conformado por una escala descriptiva de cuatro opciones; a mayor puntuación, mayor gravedad de la sintomatología. El informante valora la gravedad de cada síntoma durante las dos semanas inmediatas previas. La puntuación total puede oscilar entre 0 y 63 . En esta investigación, la escala presentó una confiabilidad alfa de Cronbach de .88 (estudio 1) y de .89 (estudio 2).

\section{Procedimiento}

Con la anuencia de las autoridades correspondientes, estudiantes de la carrera de psicología previamente entrenados aplicaron los cuestionarios en el salón de clase de cada grupo escolar, en sesiones de 35 a 45 minutos aproximadamente. Se pidió a los participantes que cumplimentaran los instrumentos de manera voluntaria y anónima.

\section{Análisis de datos}

Mediante el programa EQS 6.2 se realizaron dos AFC sobre la matriz de correlaciones policóricas, en busca de datos sobre el ajuste de los dos modelos: unidimensional (muestra 1) y de dos factores (muestra 2). Se examinó el ajuste de cada modelo en términos de la chi cuadrada escalada de Satorra-Bentler y el error cuadrático medio de aproximación (RMSEA), así como de los índices de ajuste comparativo (CFI), incremental (IFI), normalizado (NFI) y no normalizado (NNFI). De acuerdo con criterios convencionales, los valores adecuados son RMSEA $\leq .06$, CFI $\geq .95, \mathrm{IFI} \geq 0.95, \mathrm{NFI}>.90$ y NNFI $\geq 0.95$ (Hu y Bentler, 1999; Kline, 2005). En caso de que la chi cuadrada resultara sesgada por el tamaño de la muestra, se calculó el cociente $\chi^{2} / g l$, el cual reduce la sensibilidad de la chi cuadrada, cuyo valor debe ser menor que 3 según Kline. Para comparar el ajuste entre los modelos se calculó el índice esperado de validación cruzada (ECVI, por sus siglas en inglés; Browne y Cudeck, 1993).

Los análisis de invarianza se realizaron entre grupos de sexo con los datos del modelo que presentó un mejor ajuste. Se examinó la invarianza mediante una serie de pruebas de restricción progresiva. Se consideraron cuatro versiones del modelo con mejor ajuste de acuerdo con los AFC iniciales: modelo 0 , sin parámetros constreñidos; modelo 1 , constreñido en cargas factoriales; modelo 2 , constreñido en correlaciones entre factores; modelo 3, constreñido tanto en las cargas factoriales como en las correlaciones entre factores.

\section{Resultados}

Los valores para el modelo unidimensional fueron aceptables sólo en dos índices $\left(n_{1}=495\right.$, tras eliminar los casos con datos faltantes; $\chi^{2} / g l=1.97$, RMSEA $=.04$ (IC $90 \%[.03, .05]), \mathrm{CFI}=.86, \mathrm{IFI}=0.86, \mathrm{NFI}=.75, \mathrm{NNFI}=$ 0.84; ver la Tabla 1). Los AFC revelaron valores aceptables en todos los índices (excepto el NFI) para el modelo de dos factores $\left(n_{2}=492\right.$, tras eliminar los casos con datos faltantes; $\chi^{2} / g l=1.52$, RMSEA $=.03($ IC $90 \%[.02, .04])$, CFI $=$ $.94, \mathrm{IFI}=0.94, \mathrm{NFI}=.85, \mathrm{NNFI}=0.94)$. El modelo de dos factores ajustó mejor que el unidimensional $(\triangle \mathrm{ECVI}=0.17)$.

Por consiguiente, el análisis de invarianza entre sexos se realizó con los datos del modelo de dos factores $\left(n_{2}=273\right.$ mujeres y 219 hombres). En los AFC de grupos múltiples, los modelos mostraron un ajuste aceptable. El modelo sin constricciones (modelo 0 ) fue equivalente a los modelos con constricciones (modelos $1-3 ; \Delta \mathrm{CFI}=.00$; ver la Tabla 2 ).

Se realizó una réplica del estudio 1 con el propósito de encontrar evidencia más contundente sobre el ajuste de los modelos unidimensional y de dos factores, así como examinar la invarianza entre sexos del modelo que presentara un mejor ajuste.

\section{Estudio 2}

\section{Método}

\section{Participantes}

Participaron 610 estudiantes (342 mujeres y 268 hombres) de educación secundaria inferior y superior (Instituto de Estadística de la UNESCO, 1997) matriculados en dos escuelas públicas, una secundaria inferior y una secundaria superior ubicadas en la Ciudad de México. Se tomó como unidad de muestreo al grupo escolar de adscripción.

El total de los participantes se dividió aleatoriamente en dos. La muestra $1(n=305)$ contaba con 176 mujeres y 129 hombres, de los cuales 155 eran estudiantes de secundaria 
Tabla 1. Cargas factoriales para los reactivos del BDI-II.

\begin{tabular}{|c|c|c|c|c|c|c|c|c|c|c|c|c|}
\hline \multirow{3}{*}{$\begin{array}{l}\text { Reactivo } \\
\text { Factor cognitivo-afectivo }\end{array}$} & \multicolumn{6}{|c|}{ Primer estudio } & \multicolumn{6}{|c|}{ Segundo estudio } \\
\hline & \multicolumn{3}{|c|}{ Modelo unidimensional } & \multicolumn{3}{|c|}{$\begin{array}{l}\text { Modelo de dos } \\
\text { factores }\end{array}$} & \multicolumn{3}{|c|}{$\begin{array}{c}\text { Modelo } \\
\text { unidimensional }\end{array}$} & \multicolumn{3}{|c|}{ Modelo de dos factores } \\
\hline & $\Lambda$ & $\mathrm{E}$ & $\mathrm{R}^{2}$ & $\Lambda$ & $\mathrm{E}$ & $\mathrm{R}^{2}$ & $\Lambda$ & $\mathrm{E}$ & $\mathrm{R}^{2}$ & $\Lambda$ & $\mathrm{E}$ & $\mathrm{R}^{2}$ \\
\hline 1. Tristeza & .59 & .80 & .35 & .61 & .78 & .37 & .55 & .82 & .31 & .67 & .73 & .46 \\
\hline 2. Pesimismo & .56 & .82 & .31 & .48 & .87 & .23 & .57 & .81 & .33 & .54 & .84 & .29 \\
\hline 3. Fracasos pasados & .60 & .80 & .36 & .60 & .79 & .36 & .64 & .76 & .41 & .66 & .74 & .44 \\
\hline 4. Pérdida del placer & .46 & .88 & .21 & .53 & .84 & .28 & .55 & .83 & .31 & .51 & .85 & .26 \\
\hline 5. Sentimientos de culpa & .50 & .86 & .25 & .51 & .85 & .26 & .65 & .76 & .42 & .59 & .80 & .35 \\
\hline 6. Sentimientos de castigo & .40 & .91 & .16 & .47 & .88 & .22 & .54 & .83 & .29 & .42 & .90 & .17 \\
\hline 7. Auto-insatisfacción & .57 & .81 & .33 & .64 & .76 & .41 & .67 & .74 & .44 & .58 & .80 & .34 \\
\hline 8. Autocrítica & .48 & .87 & .23 & .61 & .79 & .37 & .53 & .84 & .28 & .58 & .81 & .33 \\
\hline 9. Pensamientos o deseos suicidas & .57 & .82 & .32 & .51 & .85 & .26 & .56 & .82 & .31 & .64 & .76 & .41 \\
\hline 10. Llanto & .48 & .87 & .23 & .59 & .80 & .35 & .51 & .85 & .26 & .54 & .83 & .29 \\
\hline 11. Agitación & .39 & .91 & .15 & .37 & .92 & .14 & .46 & .88 & .21 & .41 & .91 & .17 \\
\hline 12. Pérdida de interés & .46 & .88 & .21 & .53 & .84 & .28 & .50 & .86 & .25 & .60 & .78 & .36 \\
\hline 13. Indecisión & .58 & .81 & .34 & .58 & .81 & .33 & .50 & .86 & .25 & .56 & .82 & .31 \\
\hline 14. Falta de valía & .62 & .78 & .38 & .67 & .73 & .45 & .58 & .81 & .34 & .62 & .78 & .38 \\
\hline 17. Irritabilidad & .53 & .84 & .28 & .56 & .82 & .32 & .55 & .83 & .30 & .62 & .77 & .39 \\
\hline 21. Pérdida de interés en el sexo & .30 & .95 & .09 & .20 & .98 & .04 & .30 & .95 & .09 & .20 & .97 & .04 \\
\hline \multicolumn{13}{|l|}{ Factor somático-vegetativo } \\
\hline 15. Pérdida de energía & .57 & .81 & .32 & .66 & .74 & .44 & .56 & .82 & .32 & .65 & .75 & .42 \\
\hline 16. Cambios en el patrón de sueño & .37 & .92 & .14 & .57 & .81 & .33 & .44 & .89 & .20 & .47 & .88 & .22 \\
\hline 18. Cambios en el apetito & .51 & .86 & .26 & .56 & .82 & .32 & .48 & .87 & .23 & .52 & .85 & .27 \\
\hline 19. Dificultad en la concentración & .48 & .87 & .23 & .61 & .79 & .37 & .49 & .86 & .24 & .61 & .79 & .37 \\
\hline 20. Cansancio o fatiga & .47 & .87 & .22 & .66 & .74 & .44 & .60 & .79 & .37 & .62 & .78 & .38 \\
\hline
\end{tabular}

Nota: $\Lambda=$ carga factorial; $E=$ error; $R^{2}=$ correlación múltiple cuadrada.

inferior y 150 de secundaria superior. La media de edad fue de 15.28 años $(D E=1.62)$. En la muestra $2(n=305)$ participaron 166 mujeres y 139 hombres (164 de secundaria inferior y 141 de secundaria superior), siendo su edad promedio de 15.12 años $(D E=1.61)$. En ambas muestras, la edad osciló entre los 13 y los 18 años.

\section{Instrumento, procedimiento y análisis de datos}

El instrumento, el procedimiento y el análisis de datos del segundo estudio fueron idénticos a los del primero, por tratarse de una réplica.

\section{Resultados}

Los AFC revelaron valores aceptables en casi todos los índices, tanto para el modelo unidimensional $\left(n_{1}=305 ; \chi^{2}\right.$ $/ g l=1.55$; RMSEA = .04, IC 90\% $[.03, .05]$; CFI = .90; $\mathrm{IFI}=0.91$; NFI $=.77$; NNFI $=0.89$; ver la Tabla 1 ) como para el de dos factores $\left(n_{2}=305 ; \chi^{2} / g l=1.14\right.$; RMSEA $=$ .02 , IC 90\% $[.03, .05]$; CFI $=.97$; IFI $=0.98$; NFI $=.83$; NNFI $=0.97)$. El modelo de dos factores arrojó un mejor ajuste $(\triangle \mathrm{ECVI}=0.24)$. 
Tabla 2. Análisis de invarianza del modelo de dos factores del BDI-II entre sexos (estudios 1 y 2).

\begin{tabular}{|c|c|c|c|c|c|c|}
\hline Modelo con constricciones en & $\chi^{2} / g l$ & $\begin{array}{l}\text { RMSEA } \\
\text { [IC 90\%] }\end{array}$ & CFI & IFI & NFI & NNFI \\
\hline \multicolumn{7}{|l|}{$\begin{array}{l}\text { Estudio } 1 \\
(n=492)\end{array}$} \\
\hline 0. Ningún parámetro & 1.12 & $\begin{array}{c}.02 \\
{[.00, .03]}\end{array}$ & .99 & 0.99 & .96 & 0.99 \\
\hline 1. Cargas factoriales & 1.14 & $\begin{array}{c}.02 \\
{[.01, .03]}\end{array}$ & .99 & 0.99 & .95 & 0.99 \\
\hline 2. Correlaciones entre factores & 1.05 & $\begin{array}{c}.01 \\
{[.00, .03]}\end{array}$ & .99 & 0.99 & .97 & 0.99 \\
\hline 3. Ambos parámetros & 1.14 & $\begin{array}{c}.02 \\
{[.01, .03]}\end{array}$ & .99 & 0.99 & .96 & 0.99 \\
\hline \multicolumn{7}{|l|}{$\begin{array}{l}\text { Estudio } 2 \\
(n=305)\end{array}$} \\
\hline 0. Ningún parámetro & 0.94 & $\begin{array}{c}.00 \\
{[.00, .02]}\end{array}$ & 1.00 & 1.00 & .94 & 1.00 \\
\hline 1. Cargas factoriales & 0.92 & $\begin{array}{c}.00 \\
{[.00, .02]}\end{array}$ & 1.00 & 1.00 & .94 & 1.00 \\
\hline 2. Correlaciones entre factores & 0.92 & $\begin{array}{c}.00 \\
{[.00, .02]}\end{array}$ & 1.00 & 1.00 & .95 & 1.00 \\
\hline 3. Ambos parámetros & 0.94 & $\begin{array}{c}.00 \\
{[.00, .02]}\end{array}$ & 1.00 & 1.00 & .94 & 1.00 \\
\hline
\end{tabular}

Nota: $\chi^{2} / g l=$ cociente chi cuadrada entre grados de libertad; RMSEA = error cuadrático medio de aproximación; CFI = índice de ajuste comparativo; IFI = índice de ajuste incremental; NFI = índice de ajuste normalizado; NNFI = índice de ajuste no normalizado.

El análisis de invarianza se llevó a cabo con los datos del modelo de dos factores, por presentar el ajuste más adecuado ( $n_{2}=166$ mujeres y 139 hombres). El ajuste de los modelos fue aceptable en los AFC de grupos múltiples. El modelo sin constricciones (modelo 0 ) fue equivalente a los modelos con constricciones (modelos 1-3; $\Delta \mathrm{CFI}=.00$; ver la Tabla 2).

\section{Discusión}

El primer propósito de esta investigación fue someter a prueba, en dos estudios independientes, dos modelos de medida de la depresión, la estructura unidimensional encontrada en Chile (Araya et al. 2013; Melipillán et al. 2008) y el modelo original propuesto por Beck et al. (1996) de los factores cognitivo-afectivo y somático-vegetativo, mediante la aplicación a adolescentes de la versión en español del BDI-II.

$\mathrm{Si}$ bien es cierto que existen distintas versiones del BDI-II para adolescentes que cuentan con propiedades psicométricas adecuadas, son escasas las que han sometido a prueba su estructura factorial. El hallazgo fundamental de la presente investigación es que el modelo original de Beck et al. (1996) de dos factores presenta mejor ajuste tanto en un primer estudio como en su réplica, en comparación con la estructura unidimensional. Este resultado sugiere que no se cumple la primera hipótesis de investigación, la cual planteaba que el modelo de una dimensión representaría la estructura factorial más adecuada, tal y como se había hallado en otras muestras latinoamericanas (Araya et al. 2013; Melipillán et al. 2008).

Para explicar la discrepancia entre los hallazgos de Chile y México, es importante señalar que los estudios chilenos no sometieron a prueba la estructura hallada por Beck et al. (1996). Araya et al. (2013) únicamente evaluaron mediante AFC el modelo unidimensional, resultado de un análisis factorial exploratorio. Por su parte, Melipillán et al. (2008) sí examinaron con análisis factorial confirmatorio dos modelos, uno unidimensional y uno de dos factores (llamados cognitivo-afectivo y somático), pero este último fue el producto de un análisis factorial exploratorio y no coincidía con el modelo propuesto por Beck et al. para población general. Las diferencias entre la estructura que encontraron y el modelo de Beck et al. radican en el arreglo factorial, donde algunos síntomas cognitivo-afectivos (reactivos 
$4,11,12,17$ y 21) se agruparon como somáticos, lo cual discrepa del planteamiento inicial. El hallazgo del modelo de dos factores de Beck et al. se ha replicado parcialmente en otros estudios (Osman et al., 2008; Wu y Chang, 2008) debido a las diferencias entre el tipo de población de estudio, ya sea clínica o general (Beck et al. 1996).

Otra posible interpretación de estos hallazgos es que la depresión, único factor del modelo unidimensional, constituya una dimensión supra-ordenada a ambos dominios sintomáticos considerados en el modelo de dos factores: el cognitivo-afectivo y el somático. De ser el caso, la depresión sería un fenómeno emergente, es decir, uno que cuenta con propiedades únicas que no se reducen a las de sus componentes. Byrne, Stewart, Kennard y Lee (2007) obtuvieron evidencia transcultural para un modelo de dos órdenes como éste. Sin embargo, los factores de primer orden de su modelo eran tres. En estudios futuros será conveniente: 1) evaluar a la luz de la teoría y evidencia sobre la depresión si estas dos interpretaciones conciliadoras son viables y, en el caso de que ambas lo fueran, 2) averiguar cuál es la mejor interpretación, comparando el ajuste de todos estos modelos: el unidimensional (Araya et al. 2013; Melipillán et al. 2008), el de dos factores (Beck et al. 1996) y el de una dimensión de segundo orden que engloba a dos de primer orden (similar al de Byrne et al. 2007).

El hecho de que en este estudio los indicadores se hayan agrupado en dimensiones teóricamente congruentes fortalece la validez de la postura de Beck acerca de la depresión, que se explica en términos de la interacción de tres componentes, a saber: 1) la triada cognitiva, que es la visión negativa de sí mismo, del mundo y del futuro, 2) los esquemas o patrones cognitivos rígidos relativamente estables que permean la manera de reaccionar cognitiva y conductualmente y 3 ) los errores del procesamiento de la información o distorsiones sistemáticas del pensamiento que validan las creencias catastróficas de la persona a pesar de carecer de evidencia que las respalde (Beck et al. 1979; Clark y Beck, 2010). Estos componentes, según Beck, son los responsables del inicio y mantenimiento de los síntomas depresivos, que se agrupan en dos dimensiones claramente discernibles: 1) cognitiva-afectiva (con ítems como tristeza, fracaso, sentimientos de culpa, sentimientos de castigo, etc.) y somático-vegetativa (p.ej., pérdida de energía, cambios en el apetito y cansancio o fatiga).

Como segundo propósito se indagó acerca de la invarianza entre sexos del modelo que presentara mejor ajuste.
Los resultados del presente estudio apoyan la segunda hipótesis, pues el modelo de dos factores no varió entre mujeres y hombres en el estudio 1 así como en el 2, lo cual implica que el constructo depresión mantiene una misma estructura en ambos sexos. Este resultado es congruente con el de Araya et al. (2013), quienes tampoco hallaron diferencias entre sexos al someter a prueba la invarianza del modelo de la depresión.

En distintas investigaciones se ha encontrado consistentemente que las mujeres presentan durante la adolescencia síntomas de depresión en mayor grado que los hombres (Araya et al. 2013; Benjet et al. 2009; Del Barrio et al. 2004; Uslu et al. 2008). También se sabe que estas diferencias, si bien existen desde el inicio de la adolescencia, continúan acentuándose conforme se aproxima el final de ésta (Hankin, 2006). No obstante, los resultados del presente estudio sugieren que dichas diferencias son cuantitativas, pero no cualitativas. La discrepancia entre este resultado y el de $\mathrm{Wu}$ (2010a) en adolescentes de Taiwán se puede adjudicar a las diferencias culturales (Wu, 2010b). Aunque generalmente son más altos los índices de depresión de las mujeres, la estructura del constructo llamado depresión es similar para ambos sexos.

La principal fortaleza de esta investigación radica en el uso de la réplica, que arrojó los mismos resultados en dos diferentes estudios mediante dos muestras independientes de adolescentes provenientes de distintas escuelas. El hecho de que el modelo de Beck et al. (1996) sea el de mejor ajuste en ambas, añade generalidad a los resultados. Como limitaciones se señala que en el presente estudio no se investigó el ajuste de los modelos factoriales en adolescentes de población clínica. Es necesario que en estudios subsiguientes se atienda esta advertencia, ya que las principales diferencias entre los modelos en la literatura internacional se han explicado en términos del tipo de población que se estudia (Beck et al. 1996; Ward, 2006).

A pesar de que el BDI-II ha mostrado buenas propiedades psicométricas a nivel mundial, es menester arrojar más luz sobre su validez y confiabilidad, así como indagar si logra discriminar entre población clínica y no clínica, con el propósito de consolidarlo como una medida adecuada para evaluar los síntomas de depresión en adolescentes. Los resultados de la presente investigación muestran evidencia en favor de la validez de constructo del BDI-II y, por lo tanto, del planteamiento teórico de Beck et al. (1996) sobre la depresión en adolescentes mexicanos. 


\section{Referencias}

Abramson, L. Y., Seligman, M. E. P. y Teasdale, J. D. (1978). Learned helplessness in humans: Critique and reformulation. Journal of Abnormal Psychology, 87, 49-74. doi:10.1037/0021-843X.87.1.49.

Agudelo, D., Spielberg, C. D. y Buela-Casal, G. (2007). La depresión: ¿Un trastorno dimensional o categorial? Salud Mental, 30(3), 20-28.

Araya, R., Montero-Marín, J., Barroilhet, S., Fritsch, R., Gaete, J. y Montgomery, A. (2013). Detecting depression among adolescents in Santiago, Chile: Sex differences. BMC Psychiatry, 13, 122 y 269. doi:10.1186/1471-244X-13-122 y 10.1186/1471-244X-13-269.

Beck, A. (1967). Diagnóstico y tratamiento de la depresión. E.U.: Merck Sharp \& Dohme International.

Beck, A. T., Rush, A. J., Shaw, B. F. y Emery, G. (1979). Cognitive therapy for depression. Nueva York, E.U.: The Guilford Press.

Beck, A. T., Steer, R. A. y Brown, G.K. (1996). Beck Depression Inventory (Second edition) [Inventario de depresión de Beck (segunda edición)] San Antonio, TX, E.U.: Psychological Corporation.

Beltrán, M. C., Freyre, M.-Á. y Hernández-Guzmán, L. (2012). El Inventario de depresión de Beck: Su validez en población adolescente. Terapia Psicológica, 30, 5-13.

Benjet, C., Borges, G., Medina-Mora, M. E., Fleiz-Bautista, C. y Zambrano-Ruiz, J. (2004). La depresión con inicio temprano: Prevalencia, curso natural y latencia para buscar tratamiento. Salud Pública de México, 46, 417-424.

Benjet, C., Borges, G., Medina-Mora, M. E., Méndez, E., Fleiz, C., Rojas, E. y Cruz, C. (2009). Diferencias de sexo en la prevalencia y severidad de trastornos psiquiátricos en adolescentes de la Ciudad de México. Salud Mental, 31, 155-163.

Brenlla, E. y Rodríguez, C. (2006). Adaptación argentina del Inventario de Depresión de Beck. Buenos Aires, Argentina: Paidós.

Browne, M. W. y Cudeck, R. (1993). Alternative ways of assessing model fit. En K. A. Bollen y J. S. Long. (Eds.). Testing structural equation models (pp. 136-162). Beverly Hills, CA, E.U.: Sage.

Butler, A. C., Chapman, J. E., Forman, E. M. y Beck, A. T. (2006). The empirical status of cognitive-behavioral therapy: A review of meta-analyses. Clinical Psychology Review, 26, 17-31. doi:10.1016/j. cpr.2005.07.003.

Byrne, B. M., Stewart, S. M., Kennard, B. D. y Lee, P. W. H. (2007). The Beck Depression Inventory-II: Testing for measurement equivalence and factor mean differences across Hong Kong and American adolescents. International Journal of Testing, 7, 293-309. doi: $10.1080 / 15305050701438058$.

Byrne, B. M., Stewart, S. M. y Lee, P. W. H. (2004). Validating the Beck Depression Inventory-II for Hong Kong community adolescents. International Journal of Testing, 4, 199-216. doi:10.1207/ s15327574ijt0403_1.

Clark, D. A. y Beck, A. T. (2010). Cognitive theory and therapy of anxiety and depression: Convergence with neurobiological findings. Trends in Cognitive Sciences, 14, 418-424. doi:10.1016/j.tics.2010.06.007.

Del Barrio, M. V., Mestre, M. V., Tur, A. M. y Samper, P. (2004). La depresión infanto-juvenil. El efecto de los factores emocionales, comportamentales y sociodemográficos. Revista de Psicología General y Aplicada, 57, 5-20

DeRubeis, R. J., Hollon, S. D., Amsterdam, J. D., Shelton, R. C., Young, P. R., Salomon, R. M.,... Gallop, R. (2005). Cognitive therapy vs medications in the treatment of moderate to severe depression. Archives of General Psychiatry, 62, 409-416. doi:10.1001/archpsyc.62.4.409.

Dobson, K. S., Hollon, S. D., Dimidjian, S., Schmaling, K. B., Kohlenberg, R. J., Gallop, R. J., Jacobson, N. S. (2008). Randomized trial of behavioral activation, cognitive therapy, and antidepressant medication in the prevention of relapse and recurrence in major depression. Journal of Consulting and Clinical Psychology, 76, 468-477. doi:10.1037/0022006X.76.3.468.

Ezquiaga T., E., García L., A., Díaz N., M. y García B., M. J. (2011). “Depresión”. Imprecisión diagnóstica y terapéutica. Importantes consecuen- cias en la práctica clínica. Revista de la Asociación Española de Neuropsiquiatría, 31, 457-475. doi:10.4321/S0211-57352011000300005.

Hankin, B. L. (2006). Adolescent depression: Description, causes, and interventions. Epilepsy \& Behavior, 8, 102-114. doi:10.1016/j. yebeh.2005.10.012.

Hernández-Guzmán, L. (2012). Informe final del proyecto IN304610 [informe interno]. Programa de Apoyo a Proyectos de Investigación e Innovación Tecnológica, Dirección General de Asuntos del Personal Académico y Facultad de Psicología, Universidad Nacional Autónoma de México. Recuperable a solicitud en http://www.transparencia.unam. $\mathrm{mx} /$ Contacto.HTM.

Hu, L. y Bentler, P. M. (1999). Cutoff criteria for fit indexes in covariance structure analysis: Conventional criteria versus new alternatives. Structural Equation Modeling: A Multidisciplinary Journal, 6, 1-55. doi:10.1080/10705519909540118.

Instituto de Estadística de la UNESCO (1997). International Standard Classification of Education mapping of national educational programmes: Mexico [hoja de cálculo electrónica]. Recuperado desde http:// www.uis.unesco.org/Education/ISCEDMappings/Pages/default.aspx.

Keenan-Miller, D., Hammen, C. L. y Brennan, P. A. (2007). Health outcomes related to early adolescent depression. Journal of Adolescent Health, 41, 256-262. doi:10.1016/j.jadohealth.2007.03.015.

Kline, R. B. (2005). Principles and practice of structural equation modeling. Nueva York, NY, E.U.: The Guilford Press.

Lakdawalla, Z., Hankin, B. L. y Mermelstein, R. (2007). Cognitive theories of depression in children and adolescents: A conceptual and quantitative review. Clinical Child and Family Psychology Review, 10, 1-24. doi:10.1007/s10567-006-0013-1.

Melipillán A., R., Cova S., F., Rincón G., P. y Valdivia P., M. (2008). Propiedades psicométricas del Inventario de depresión de Beck-II en adolescentes chilenos. Terapia Psicológica, 26, 59-69.

Naicker, K., Galambos, N. L., Zeng, Y., Senthilselvan, A. y Colman, I. (2013). Social, demographic, and health outcomes in the 10 years following adolescent depression. Journal of Adolescent Health, 52, 533-538. doi:10.1016/j.jadohealth.2012.12.016.

Nolen-Hoeksema, S. (1991). Responses to depression and their effects on the duration of depressive episodes. Journal of Abnormal Psychology, 100, 569-582. doi:10.1037/0021-843X.100.4.569.

Organización Mundial de la Salud (2012). La depresión (nota descriptiva No. 369). Recuperado desde http://www.who.int/mediacentre/ factsheets/fs369/es/.

Organización Panamericana de la Salud (2008). Jóvenes: Opciones y cambios. EU: Organización Mundial de la Salud.

Osman, A., Barrios, F. X., Gutierrez, P. M., Williams, J. E., y Bailey, J. (2008). Psychometric properties of the Beck Depression Inventory-II in nonclinical adolescent samples. Journal of Clinical Psychology, 64, 83-102. doi:10.1002/jclp.20433.

Osman, A., Downs, W. R., Barrios, F. X., Kopper, B. A., Gutierrez, P. M., y Chiros, C. E. (1997). Factor structure and psychometric characteristics of the Beck Depression Inventory-II. Journal of Psychopathology and Behavioral Assessment, 19, 359-376. doi:10.1007/BF02229026.

Sandín, B. (2003). Escalas PANAS de afecto positivo y negativo para niños y adolescentes (PANASN). Revista de Psicopatología y Psicología Clínica, 8, 173-182. doi:10.5944/rppc.vol.8.num.2.2003.3953.

Uslu, R. I., Kapci, E. G., Oncu, B., Ugurlu, M. y Turkcapar, H. (2008). Psychometric properties and cut-off scores of the Beck Depression Inventory-II in Turkish adolescents. Journal of Clinical Psychology in Medical Settings, 15, 225-233. doi:10.1007/s10880-008-9122-y.

Valladares, A., Dilla, T. y Sacristán, J. A. (2009). La depresión: Una hipoteca social. Últimos avances en el conocimiento del coste de la enfermedad. Actas Españolas de Psiquiatría, 37, 49-53.

VanVoorhis, C. R. W. y Blumentritt, T. L. (2007). Psychometric properties of the Beck Depression Inventory-II in a clinically-identified sample of Mexican American adolescents. Journal of Child and Family Studies, 16, 789-798. doi:10.1007/s10826-006-9125-y.

Ward, L. C. (2006). Comparison of factor structure models for the Beck Depression Inventory-II. Psychological Assessment, 18, 81-88. doi:10.1037/1040-3590.18.1.81. 
Wu, P. -C. (2010a). Differential functioning of the Chinese version of Beck Depression Inventory-II in adolescent gender groups: Use of a multiple-group mean and covariance structure model. Social Indicators Research, 96, 535-550. doi:10.1007/s11205-009-9491-0.

$\mathrm{Wu}$, P. -C. (2010b). Measurement invariance and latent mean differences of the Beck Depression Inventory II across gender groups. Journal of Psychoeducational Assessment, 28, 551-563. doi: $10.1177 / 0734282909360772$.

Wu, P. -C. y Chang, L. (2008). Psychometric properties of the Chinese version of the Beck Depression Inventory-II using the Rasch model. Measurement and Evaluation in Counseling and Development, 41, 13-31.

Zauszniewski, J. A. y Rong, J.-R. (1999). Depressive cognitions and psychosocial functioning: A test of Beck's cognitive theory. Archives of Psychiatric Nursing, 13, 286-293. doi:10.1016/S0883-9417(99)80060-0. 
